\title{
A 3D motion capture analysis of a giving-way ankle episode during a 180-degree pivot turn : A case report
}

\section{Chin, Mathieu}

2021-03-30

Chin , M , Leppanen , M , Kulmala , J-P , Vasankari , T , Parkkari , J \& Pasanen , K 2021 , ' A 3D motion capture analysis of a giving-way ankle episode during a 180-degree pivot turn : A case report ' , Journal of Biomechanics , vol. 118 , 110318 . https://doi.org/10.1016/j.jbiomech.2021.110318

http://hdl.handle.net/10138/330710

https://doi.org/10.1016/j.jbiomech.2021.110318

cc_by_nc_nd

publishedVersion

Downloaded from Helda, University of Helsinki institutional repository.

This is an electronic reprint of the original article.

This reprint may differ from the original in pagination and typographic detail.

Please cite the original version. 


\title{
A 3D motion capture analysis of a giving-way ankle episode during a 180-degree pivot turn: A case report
}

\author{
Mathieu Chin ${ }^{\mathrm{a}, *}$, Mari Leppänen ${ }^{\mathrm{b}}$, Juha-Pekka Kulmala ${ }^{\mathrm{c}}$, Tommi Vasankari ${ }^{\mathrm{b}}$, Jari Parkkari ${ }^{\mathrm{b}, \mathrm{f}}$, \\ Kati Pasanen ${ }^{\text {a,b,d,e }}$
}

a Sports Injury Prevention Research Centre, Faculty of Kinesiology, University of Calgary, Calgary, Canada

${ }^{\mathrm{b}}$ Tampere Research Centre of Sports Medicine, UKK Institute, Tampere, Finland

${ }^{\mathrm{c}}$ Motion Analysis Laboratory, Children's Hospital, University of Helsinki and Helsinki University Hospital, Helsinki, Finland

${ }^{\mathrm{d}}$ Alberta Children's Hospital Research Institute, University of Calgary, Calgary, Canada

e McCaig Institute for Bone and Joint Health, University of Calgary, Calgary, Canada

${ }_{\mathrm{f}}$ University of Tampere, Tampere, Finland

\section{A R T I C L E I N F O}

\section{Article history:}

Accepted 3 February 2021

\section{Keywords:}

Ankle

Giving-way

Mechanism

\begin{abstract}
A B S T R A C T
Lateral ankle sprains are highly prevalent during sporting activities, as it accounts for approximately $60 \%$ of all athletic injuries. There is currently a paucity of research which provides kinetic and kinematic assessments of sprains and giving-way episodes of the ankle joint. The aim of this case study was to examine the kinetics and kinematics of the ankle and knee during a giving-way episode in a female ice hockey player during a 180-degree pivot turn, which was conducted in a 3D motion capture laboratory. Three trials were conducted on this participant (one accident trial, two control trials). Kinetic and kinematic analysis was conducted on the outside leg during a left 180-degree pivot turn (right leg). The plantarflexion angle of the giving-way trial was larger than the control trials from the point of initial contact until the end of the trial. Inversion was also 27-degrees greater $150 \mathrm{~ms}$ after initial contact in the giving-way trial and 26-degrees greater compared to control trials 1 and 2. Substantially greater plantarflexion, inversion and internal rotation angles of the ankle were observed during the giving-way trial compared to control trials. The maximum vertical and horizontal ground reaction forces, as well as ankle inversion and internal rotation moments, were lower for the giving-way trial in comparison to the control trials. Further research is needed to understand the influence of plantarflexion angle with a giving-way episode of the ankle. This study provides valuable kinetic and kinematic information regarding a giving-way episode of the ankle.
\end{abstract}

(c) 2021 Elsevier Ltd. All rights reserved.

\section{Introduction}

Ankle injuries are extremely common in sports (Doherty et al., 2013; Fong et al., 2009). More specifically, a lateral ankle sprain (LAS) is a highly prevalent acute sports injury suffered by athletes, with approximately $60 \%$ of all athletic injuries being LAS (Wikstrom et al., 2013). Clinically, inversion trauma at the ankle joint was presented as excessive supination (Garrick, 1977). This has since been studied qualitatively (Andersen et al., 2004) and quantitatively (Fong et al., 2009), though our knowledge of the kinematics and kinetics remains limited due to the paucity of research regarding inversion trauma at the ankle joint. Often, there are short-term and long-term consequences resulting from LAS,

\footnotetext{
* Corresponding author at: 2500 University Dr NW, Calgary, AB T2N 1N4, Canada. E-mail address: mathieu.chin@ucalgary.ca (M. Chin).
}

including immediate time lost and treatment costs, and the possibility of chronic ankle instability that may lead to ankle osteoarthritis (Bahr and Krosshaug, 2005; Wikstrom et al., 2013; Vuurberg et al., 2018). Episodes of giving-way at the ankle joint are also long-term sequalaes that may lead to chronic ankle instability (Delahunt et al., 2010). As defined by Delahunt et al. (2010), a giving-way episode of the ankle joint is "the regular occurrence of uncontrolled and unpredictable episodes of excessive inversion of the rear foot (usually experienced during initial contact during walking or running), which do not result in an acute lateral ankle sprain". To reduce these injuries from occurring, there must be a better understanding of the biomechanics involved with givingway episodes during sports-related movements, allowing for various preventative measures to be incorporated (Krosshaug and Bahr, 2005; McKeon and Mattacola, 2008). 
3D Motion capture currently provides the most precise and accurate means of determining the kinetics and kinematics of sporting human movements. Giving-way incidents and injuries that occur during motion capture experiments provide valuable opportunities to quantify the kinetics and kinematics that may result in sports injuries (Krosshaug et al., 2005). Current video analysis literature using model-based image systems suggests that a sudden inversion and internal rotation of the ankle joint may be biomechanical motions leading to LAS (Panagiotakis et al., 2017; Fong et al., 2012; Mok et al., 2011).

Likewise, in a motion capture case study conducted by Kristianslund et al. (2011), the sudden inversion and internal rotation was seen to have peaked $130 \mathrm{~ms}$ to $180 \mathrm{~ms}$ after initial contact. This injury occurred during a 90-degree cutting movement. Studies by Gehring et al. (2013), Terada and Gribble (2015), and Li et al. (2019) found similar results regarding the impact of internal rotation and the sudden inversion in LAS. Case studies on giving-way episodes have been studied during a maximum effort agility T-test (Remus et al., 2018) and during a single-leg landing task (Kosik et al., 2019), which saw excessive internal rotation of the ankle joint during the incident. Despite these studies, there is currently a paucity of research which looks to quantify the kinetics and kinematics of a giving-way injury during a 180-degree pivot turn.

A better understanding of the movements associated with giving-way ankle episodes will allow for better implementation of prevention strategies that reduce ankle sprain injuries from occurring in athletes. The aim of this case study was to describe the kinematics and kinetics of a giving-way episode that occurred in a motion analysis laboratory during a 180-degree pivot turn.

\section{Methods}

The severity of the incident was minor and there was no time loss from sport for the participant. In addition, the incident was not clinically diagnosed by a physician. As such, the accident in this study was consistent with a giving-way episode of the ankle joint where there was supination during the initial contact of the forefoot (Fig. 1). The giving-way episode occurred during a baseline testing session for a cohort study (Pasanen et al., 2015) which looked to assess the risk of lower extremity injuries in youth athletes. The study protocol was approved by the Ethics Committee of Pirkanmaa Hospital District, Tampere, Finland, (ETL-code R10169). The player was an elite female ice hockey athlete $(160 \mathrm{~cm}, 57 \mathrm{~kg}, 18$ years old, dominant left foot) who had no prior injury history to the right ankle prior to the giving-way episode seen during the baseline testing session. The principal investigator/physical therapist (KP) checked the ankle following the incident, in which there were no signs of swelling or ligament rupture. The player was able to walk normally, was sore on the lat- eral aspect of her ankle, but had no other symptoms. Contacting the player afterwards, she was able to train normally the next day. The participant signed a consent form prior to their participation in this study. The giving-way episode was to the right ankle (outside leg) of the athlete during a left 180-degree pivot turn (Fig. 1).

During testing, the player wore tight shorts, a sports bra, and indoor sports shoes. 3D motion capture of the participant was conducted using eight high-speed cameras (Vicon T40, Oxford, UK) operating at $300 \mathrm{~Hz}$, along with two force platforms (AMTI, BP6001200, MA, USA) recording at $1500 \mathrm{~Hz}$. Reflective markers according to Plug-in-Gait Full Body model (Vicon, Oxford, UK) (which were placed by a trained physical therapist) were positioned on anatomical landmarks of the lower extremities. Both marker and ground reaction force (GRF) data were low-pass filtered at $15 \mathrm{~Hz}$ using a 4th order Butterworth filter. Vicon Nexus software was used to calculate kinematic and kinetic data about an orthogonal axis system located in the distal segment of a joint.

Analysis consisted of two control trials which the participant successfully completed, along with the accident trial where the giving-way episode occurred. The three trials contained the same movement where the participant performed a left 180-degree pivot turn.

All analysis was conducted on the outside leg (right leg) which made initial contact during the left 180-degree pivot turn. Analysis was consistent with a case study conducted by Kristianslund et al. (2011) where the movements were broken down into three phases (0-50 ms, 50-80 ms, and $80-170 \mathrm{~ms}$ following contact), with the first phase being the contact phase.

Outcome measures included ankle angles (degrees), knee angles (degrees), ankle moments (Nm), knee moments (Nm), and GRF (\% body weight) for the three analysed trials. Approach speed $(\mathrm{m} / \mathrm{s})$, pelvic angle (degree), center of mass (CoM) distance $(\mathrm{cm})$, measured from the participants horizontal center of mass distance to the analysis leg, and step length $(\mathrm{cm})$, distance between the participants two ankles, were also measured at the point of contact.

\section{Results}

The overall time frame used in the analysis was $67 \mathrm{~ms}$ prior to the initial contact phase by the right leg of the participant on the force plate. In the following figures, the contact phase was indicated by the time $0 \mathrm{~ms}$. Analysis followed the initial contact by $300 \mathrm{~ms}$.

Approach speed at the point of contact was $2.6 \mathrm{~m} / \mathrm{s}$ for the accident trial, $2.3 \mathrm{~m} / \mathrm{s}$ for the control 1 trial, and $2.6 \mathrm{~m} / \mathrm{s}$ for the control 2 trial. The accident trial also saw a CoM and step length distance of $52 \mathrm{~cm}$ and $51 \mathrm{~m}$, respectively. For control 1, this was $59 \mathrm{~cm}$ for both the CoM and step distance, and $61 \mathrm{~cm}$ and $65 \mathrm{~cm}$ for control 2 for the CoM and step length distance, respectively. The accident
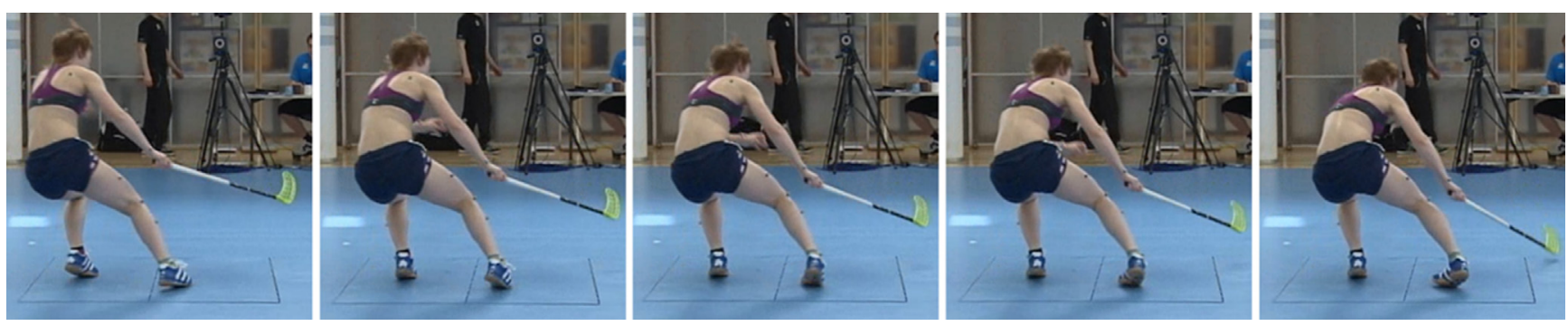

Fig. 1. Screenshots from posterior view showing the giving-way episode during a 180-degree pivot turn. 
trial had a global pelvic angle at initial contact of -36 degrees in the transverse plane. The pelvic angle was -13 degrees and -22 degrees, for controls 1 and 2, respectively.

\subsection{Ankle angles}

The maximum plantarflexion angle occurred during the accident trial at $270 \mathrm{~ms}$ from the point of initial contact with an angle of 47 degrees (Fig. 2). In contrary, the control trials both had a greater dorsiflexion angle at the point of contact, where control trials 1 and 2 respectively saw 2 - and 4-degree angles (compared to an 11-degree plantarflexion angle in the accident trial). The inversion angle of the accident trial was 22-degrees $150 \mathrm{~ms}$ after initial contact (compared to eversion angles of 4-degrees for both control trials) while the peak internal rotation angle was 50-degrees 150 ms after contact (compared to 12 and 11-degrees of external rotation for Control 1 and 2, respectively).

\subsection{Knee angles}

The minimum knee flexion angle was 40 degrees for the injury trial, whereas control 1 was 14 -degrees and control 2 was 15degrees (Fig. 2). The knee adduction angle was 2-degrees $123 \mathrm{~ms}$ after initial contact in the accident trial (abduction angles of 15degrees at $123 \mathrm{~ms}$ and 8-degrees $140 \mathrm{~ms}$ after initial contact for controls 1 and 2, respectively), while the maximum external rotation was 4-degrees at $90 \mathrm{~ms}$ after initial contact (14-degrees and 10-degrees at $36 \mathrm{~ms}$ for controls 1 and 2, respectively).

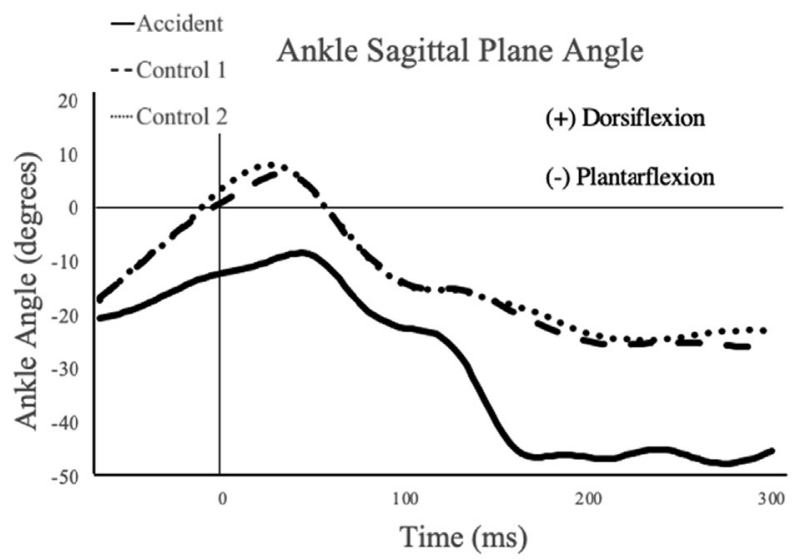

Ankle Frontal Plane Angle

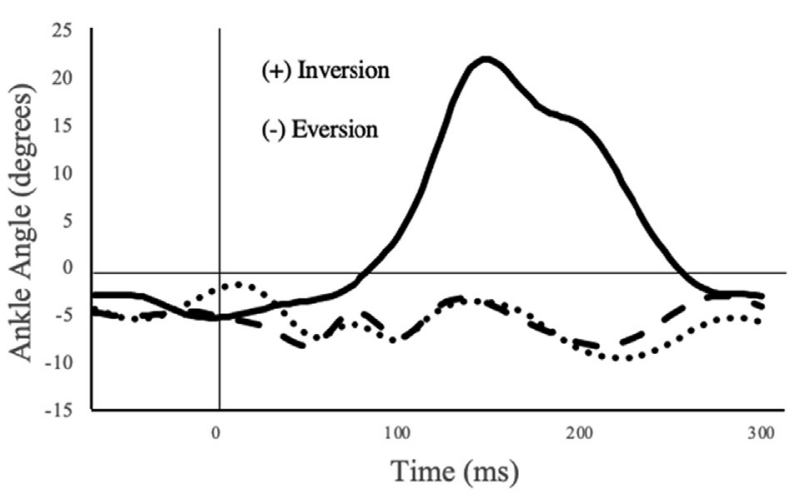

Ankle Transverse Plane Angle

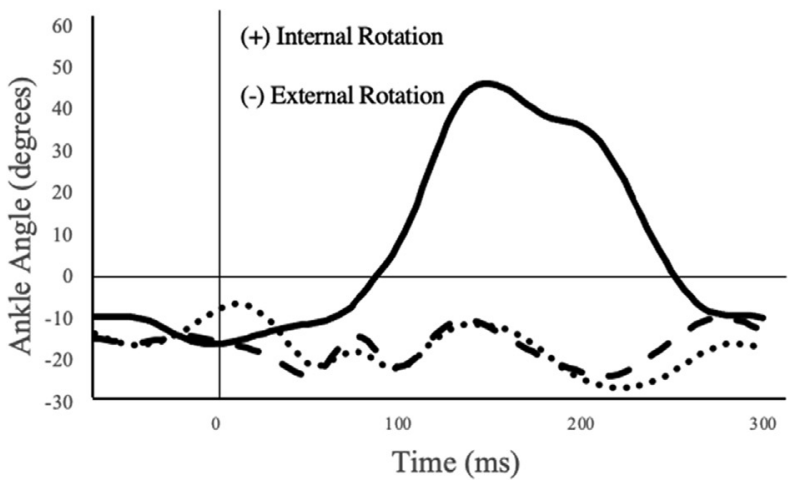

Knee Sagittal Plane Angle

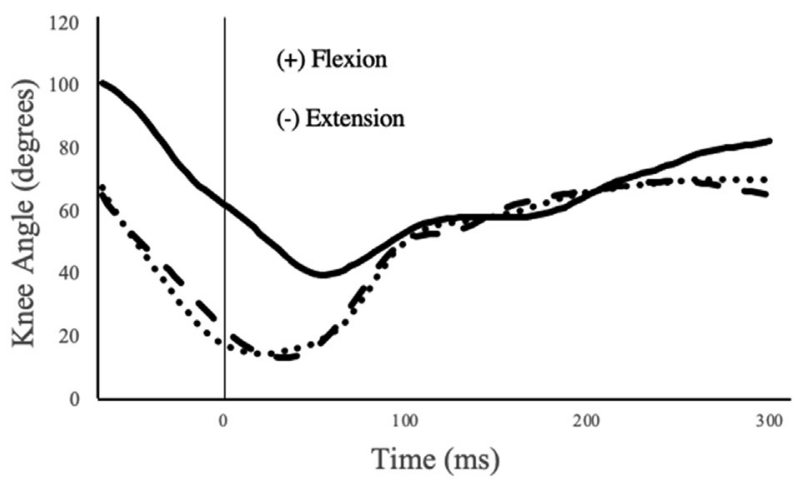

Knee Frontal Plane Angle

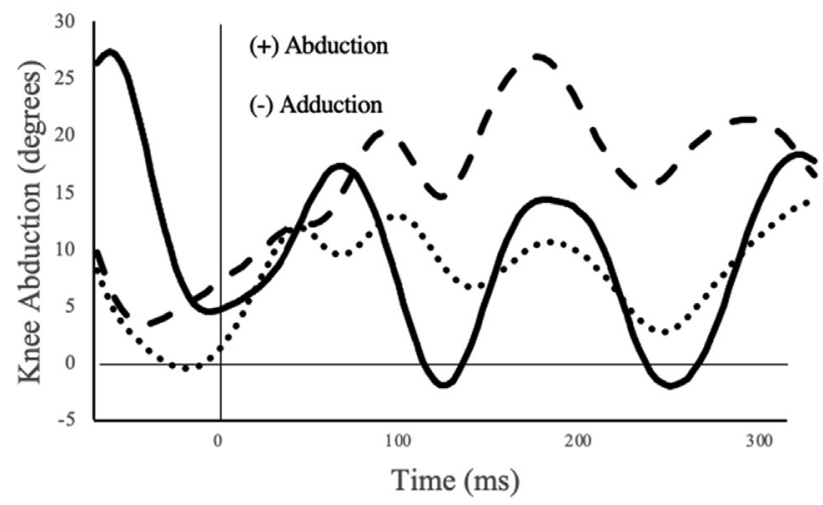

Knee Transverse Plane Angle

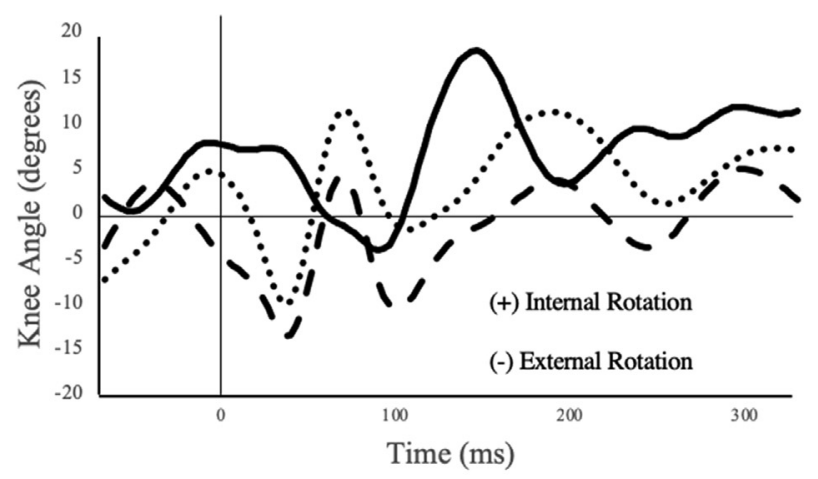

Fig. 2. Time course of ankle and knee angles (degrees) of the participant for the accident trial (solid line) and two control trials (dashed lines). Time of $0 \mathrm{~ms}$ indicates the athlete's point of initial contact. 


\subsection{Ankle moments}

The accident trial saw an ankle dorsiflexion moment of $177 \mathrm{Nm}$ at $90 \mathrm{~ms}$ after contact, compared to $154 \mathrm{Nm}$ at $90 \mathrm{~ms}$ for control 1 and $116 \mathrm{Nm}$ at $77 \mathrm{~ms}$ for control 2 (Fig. 3). The control trials had an ankle inversion moment of $65 \mathrm{Nm}$ and $51 \mathrm{Nm}$, for Trial 1 and 2, respectively, compared to $51 \mathrm{Nm}$ for the accident trial. The ankle internal rotation moment was much larger in the control trials compared to the accident trial shortly following initial contact (46 $\mathrm{Nm}$ and $43 \mathrm{Nm}$ at $63 \mathrm{~ms}$ vs $23 \mathrm{Nm}$ at $103 \mathrm{~ms}$ after contact).

\subsection{Knee moments}

The maximum knee flexion moment was $136 \mathrm{Nm}$ (at $107 \mathrm{~ms}$ after initial contact), $111 \mathrm{Nm}$ (at $103 \mathrm{~ms}$ after initial contact), and $102 \mathrm{Nm}$ (at $107 \mathrm{~ms}$ after initial contact) for the accident, con-

\section{Ankle Sagittal Plane Moment}

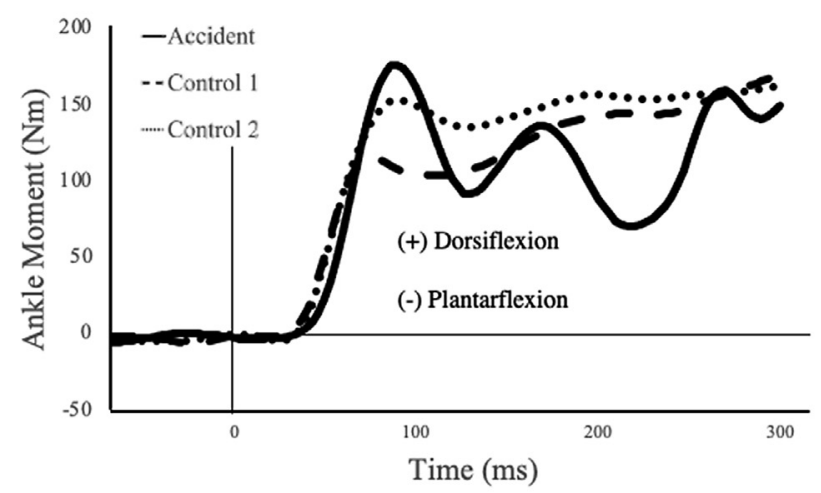

Ankle Frontal Plane Moment

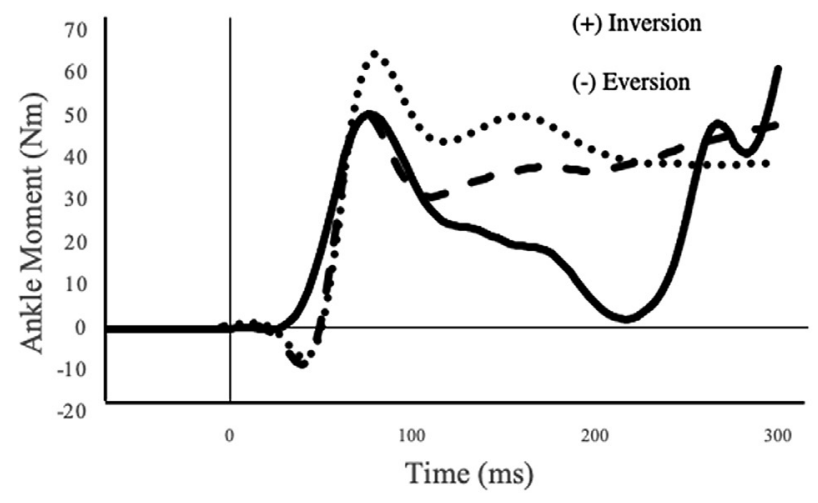

Ankle Transverse Plane Moment

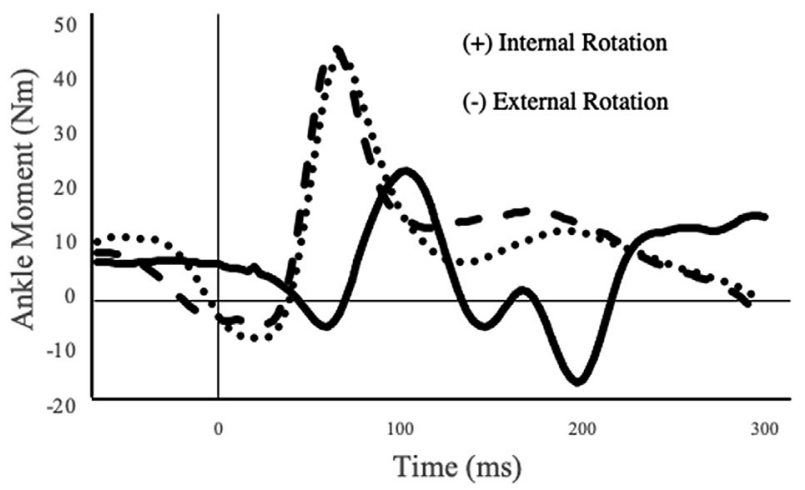

trol 1, and control 2 trials, respectively (Fig. 3). At $87 \mathrm{~ms}$ after initial contact, the knee abduction moment was $19 \mathrm{Nm}$ for the accident trial. For control 1 and 2, the knee abduction angle was $35 \mathrm{Nm}$ at $100 \mathrm{~ms}$ and $29 \mathrm{Nm}$ at $103 \mathrm{~ms}$ after initial contact, respectively. At $63 \mathrm{~ms}$ after initial contact, the accident trial had a knee internal rotation moment of $14 \mathrm{Nm}$, whereas control 1 and 2 had external rotation moments of $35 \mathrm{Nm}$ and $31 \mathrm{Nm}$, respectively.

\subsection{Ground reaction forces}

The maximum GRF for the accident trial was approximately $90 \%$ body weight and $142 \%$ body weight for the horizontal and vertical forces, respectively, which occurred $97 \mathrm{~ms}$ after the initial contact. In comparison, Control 1 had a horizontal and vertical GRF of approximately $114 \%$ and $166 \%$ body weight, respectively, whereas

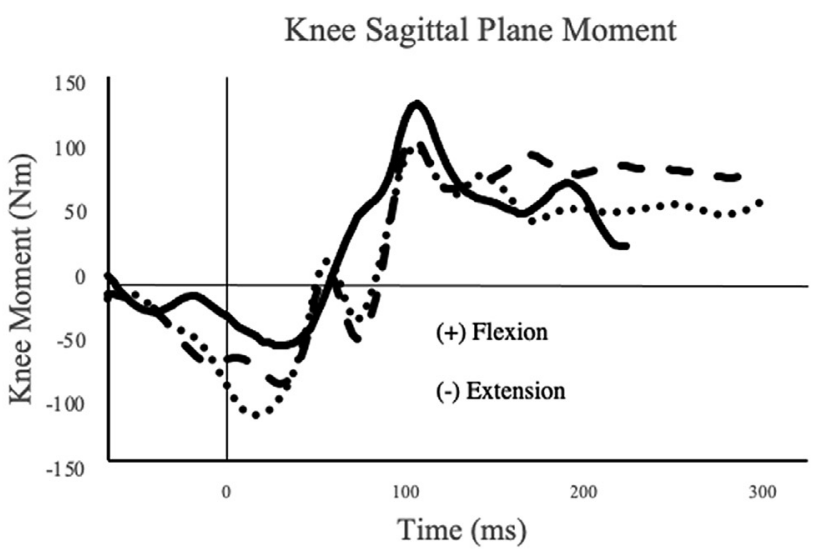

Knee Frontal Plane Moment

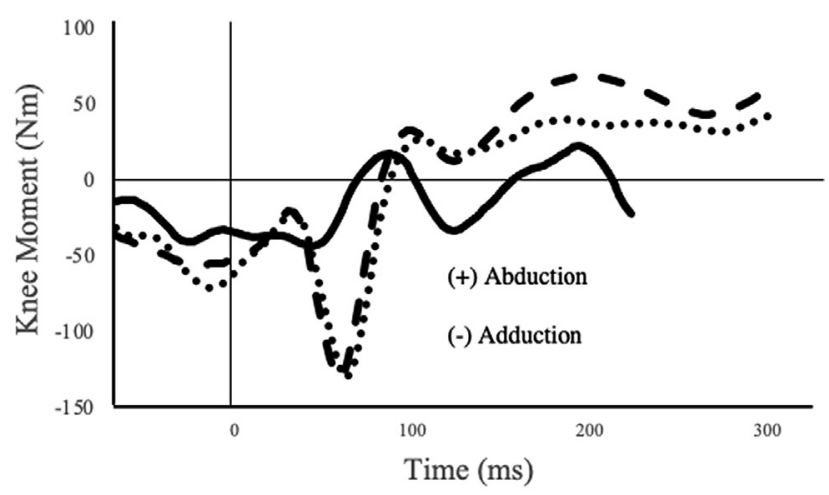

Knee Transverse Plane Moment

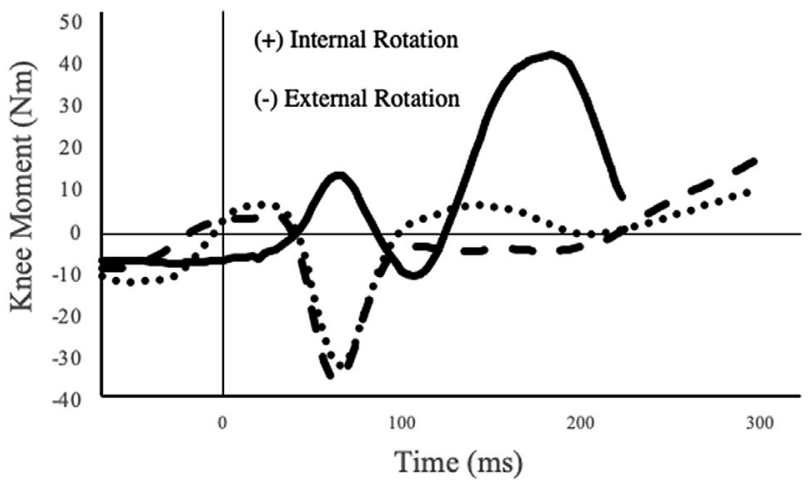

Fig. 3. Time course of ankle and knee moments $(\mathrm{Nm})$ of the participant for the accident trial and two control trials. Time of 0 ms indicates the athlete's point of initial contact. 


\section{Horizontal Ground Reaction Force}

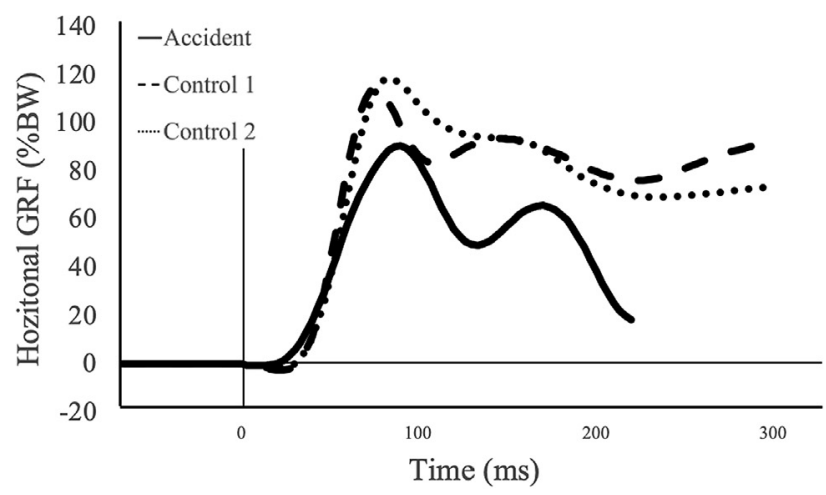

Vertical Ground Reaction Force

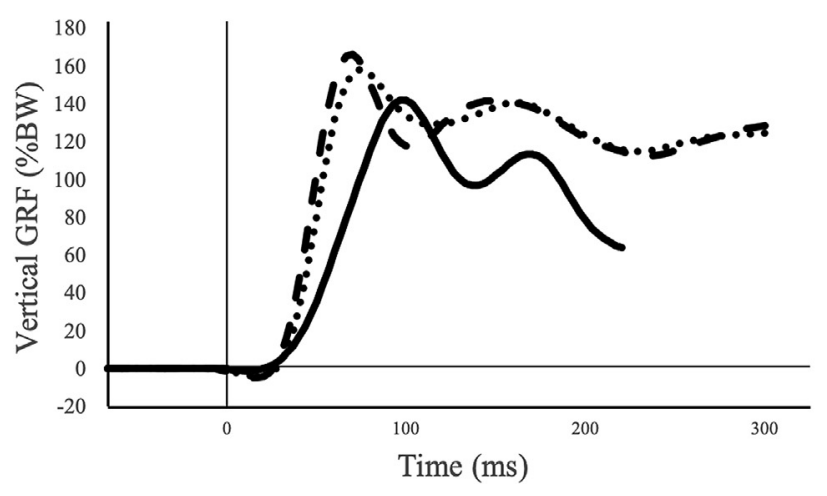

Fig. 4. Time course of the ground reaction forces $(N)$ of the participant for the accident trial and two control trials as a percentage of the participants body weight. Time of 0 ms indicates the athlete's point of initial contact.

control 2 had a horizontal and vertical GRF of approximately $118 \%$ and $158 \%$ body weight, respectively (Fig. 4 ).

\section{Discussion}

This study provides detailed kinetics and kinematics analysis of a LAS that occurred during a motion capture laboratory experiment. We notice that the maximum ankle dorsiflexion moment was greater during the accident trial compared to the two control trials shortly after initial contact. For the duration of the trial beginning at initial contact, plantarflexion was greater during the accident trial compared to the control trials. There was also a substantial greater inversion angle of the ankle joint and a greater knee adduction angle during the accident trial when compared to the control trials. The knee abduction moment was lower, and the knee flexion angle was higher after initial contact in the accident trial when compared to the control trials, whereas there was a substantial difference in the internal rotation moment shortly following initial contact. The approach speed at initial contact for the participant was similar between the three trials. The accident trial had a smaller CoM and step distance compared to the control trials as well as the pelvic alignment more towards running direction in comparison to the control trials. Both the vertical and horizontal GRF's were lower in the accident trial. Taking into consideration the different pelvic alignment and shorter CoM and step length distance, initial contact when the giving-way episode occurred may have been too early, suggesting a poor preparation that may be caused by the foot sticking to the ground. The larger knee flexion and abduction angles prior to initial contact in the accident trial compared to the control trials are also suggestive of this poor preparation. All the movements performed in the study looked to simulate game-like play to obtain the most accurate results.

These findings are consistent with those found by Kristianslund et al. (2011), where the unphysiological ankle inversion and internal rotation ankle moments occurred approximately 60-180 ms following initial contact, which resulted in high joint deflection that caused the giving-way episode (Parenteau et al., 1998). Regarding the involvement of plantar flexion angle, there has been conflicting evidence in literature. Kristianslund et al. (2011), Terada and Gribble (2015), and Fong et al. (2009) have suggested that plantar flexion does not contribute to giving-way episodes or LAS. In the contrary, studies by Kosik et al. (2019) and Gehring et al. (2013) suggested an increase in plantar flexion angle during giving-way episodes. As such, it may be beneficial for further analysis of plantarflexion in understanding the influence of plantarflexion on giving-way episodes and LAS. As ankle inversion is one of the main causes and indicators of LAS and giving-way episodes, significant results in the inversion angle are consistent with Kristianslund et al. (2011) and Kosik et al. (2019) where there was a substantially greater ankle inversion in the accident trial. This also holds true for the internal rotation of the ankle, where the results of the accident trial are consistent with literature and the angle being substantially greater than the control trials. However, Vertical GRF results from this study are contrary to those seen by Kristianslund et al. (2011), where they saw an increased force for the accident trial compared to a decrease in our study, which may explain the giving-way episode of the right ankle. This low vertical GRF may be a result of the foot sticking to the ground prior to weight acceptance of the movement to give the participant more time to reaction to the perturbation and stop the turning movement. Consequently, the leg became more compliant after initial contact, as indicated by a greater knee flexion angle and moment, which helped to decelerate CoM more slowly resulting in lower GRFs as compared to the control trials with stiffer leg behavior.

Identification and understanding of giving-way mechanisms is an essential step in sport injury prevention. Results from this study are important in differentiating normal ankle movements during a 180-degree pivot turn in comparison to movements that may increase the risk of LAS. Results of this case study may inform the development of ankle injury prevention strategies in pivoting sports.

\section{Acknowledgements}

We acknowledge the funding from Finnish Ministry of Education and Culture, Finnish Olympic Committee, and Competitive State Research Financing of the Expert Responsibility Area of Tampere University Hospital (Grants 9S047, 9T046 and 9U044). No sponsor has any role or influence in the study design, data collection, and interpretation of the data.

\section{Declaration of Competing Interest}

No authors in this study have any financial or personal relationships with any organizations or participants in the study which may influence the work.

\section{References}

Andersen, T.E., Floerenes, T.W., Arnason, A., Bahr, R., 2004. Video analysis of the mechanisms for ankle injuries in football. Am. J. Sports Med. 32, 69S-79S.

Bahr, R., Krosshaug, T., 2005. Understanding injury mechanisms: a key component of preventing injuries in sport. Br. J. Sports Med. 39 (6), 324-329. 
Delahunt, E., Coughlan, G., Caulfield, B., Nightingale, E., Lin, C., Hiller, C., 2010. Inclusion criteria when investigating insufficiencies in chronic ankle instability. Med. Sci. Sports Exerc. 42, 2106-2121.

Doherty, C., Delahunt, E., Caulfield, B., Hertel, J., Ryan, J., Bleakley, C., 2013. The incidence and prevalence of ankle sprain injury: a systematic review and metaanalysis of prospective epidemiological studies. Sports Medicine 44 (1), 123140.

Fong, D.T., Ha, S., Mok, K., Chan, C., Chan, K., 2012. Kinematics analysis of ankle inversion ligamentous sprain injuries in sports: five cases from televised tennis competitions. Am. J. Sports Med. 40 (11), 2627-2632.

Fong, D.T., Hong, Y., Shima, Y., Krosshaug, T., Yung, P.S., Chan, K.M., 2009. Biomechanics of supination ankle sprain: a case report of an accidental injury event in the laboratory. Am. J. Sports Med. 37, 822-827.

Garrick, J.G., 1977. The frequency of injury, mechanism of injury, and epidemiology of ankle sprains. Am J Sports Med 5 (6), 241-242.

Gehring, D., Wissler, S., Mornieux, G., Gollhofer, A., 2013. How to sprain your ankle a biomechanical case report of an inversion trauma. J. Biomech. 46 (1), 175-178.

Kosik, K.B., Hock, M.C., Heebner, N.R., Hartzell, J., Gribble, P.A., 2019. A laboratory captured 'giving way' episode during a single-leg landing task in an individual with unilateral chronic ankle instability. J Biomech 90, 153-158.

Kristianslund, E., Bahr, R., Krosshaug, T., 2011. Kinematics and kinetics of an accidental lateral ankle sprain. J Biomech 44 (14), 2576-2578.

Krosshaug, T., Andersen, T., Olsen, O., Myklebust, G., Bahr, R., 2005. Research approaches to describe the mechanisms of injuries in sport: Limitations and possibilities. Br J Sports Med 39 (6), 330-339.

Krosshaug, T., Bahr, R., 2005. A model-based image-matching technique for threedimensional reconstruction of human motion from uncalibrated video sequences. J Biomech 38, 919-929.

Li, Y., Ko, J., Zhang, S., Brown, C., Simpson, K., 2019. Biomechanics of ankle giving way: a case report of accidental ankle giving way during the drop landing test. J Sport Health Sci 8 (5), 494-502.
McKeon, P.O., Mattacola, C.G., 2008. Interventions for the prevention of first time and recurrent ankle sprains. Clin Sport Med 27.

Mok, K.M., Fong, D.T., Krosshaug, T., Engebretsen, L., Hung, A.S., Yung, P.S., Chan, K. M., 2011. Kinematics analysis of ankle inversion ligamentous sprain injuries in sports: 2-cases during the 2008 Beijing Olympics. Am J Sports Med 39 (7), 1548-1552.

Panagiotakis, E., Mok, K., Fong, D., Bull, A., 2017. Biomechanical analysis of ankle ligamentous sprain injury cases from televised basketball games: understanding when, how and why ligament failure occurs. J Sci Med Sport 20 (12), 1057-1061.

Parenteau, C.S., Viano, D.C., Petit, P.Y., 1998. Biomechanical properties of human cadaveric ankle-subtalar joints in quasi-static loading. J Biomech Eng 120, 105111.

Pasanen, K., Rossi, M., Parkkari, J., Heinonen, A., Steffen, K., Myklebust, G., Krosshaug, T., Vasankari, T., Kannus, P., Avela, J., Kulmala, J.P., Pertunen, J., Kujala, U.M., Bahr, R., 2015. Predictors of lower extremity injuries in team sports (PROFITS-study): a study protocol. BMJ Open Sport \& Exercise Medicine 1 (1), E000076.

Remus, A., Caulfield, B., Doherty, C., Crowe, C., Severini, G., Delahunt, E., 2018. A laboratory captured "giving way" episode in an individual with chronic ankle instability. J Biomech 76, 241-246.

Terada, M., Gribble, P., 2015. Jump landing biomechanics during a laboratory recorded recurrent ankle sprain. Foot Ankle Int 36 (7), 842-848.

Vuurberg, G., Hoorntje, A., Wink, L.M., van der Doelen, B.F.W., van den Bekerom, M. P., Dekker, R., van Dijk, C.N., Krips, R., Loogman, M.C.M., Ridderikhof, M.L., Smithuis, F.F., Stufkens, S.A.S., Verhagen, E.A.L.M., de Bie, R.A., Kerkhoffs, G.M.M. J., 2018. Diagnosis, treatment and prevention of ankle sprains: update of an evidence-based guideline. Br J Sports Med 52, 956.

Wikstrom, E., Hubbard-Turner, A., McKeon, T., 2013. Understanding and treating lateral ankle sprains and their consequences. Sports Med 43 (6), 385-393. 